\title{
LA ELABORACIÓN DE LOS CONCEPTOS CIENTÍFICOS
}

Fabio Vélez U. Ph. D.*

\begin{abstract}
One of the Lines of research of the interinstitutional Doctorat on Education of Sciences, coordinated by the Universidad Pedagogica Nacional, is titled "Elaboration of the scientific Concepts" The present article tries to explain, justify and delimitate the field of action of the Line. The article is divided in two parts. In the first one, we give an example of what we understand about such Elaboration. In the second one, we establish the theorical frame of the Lines.
\end{abstract}

\section{Introducción}

Una de las líneas de investigación del Doctorado interinstitucional de Educación en Ciencias, coordinado por la Universidad Pedagógica Nacional, lleva el nombre de "elaboración de los conceptos científicos". El presente artículo pretende explicar, justificar y delimitar el campo de acción de la línea. El articulo tiene dos partes. En la primera Ponemos un ejemplo de lo que entendemos por elaboración de los conceptos, y en la segunda presentamos el marco teórico de la línea, tomando como base los resultados del análisis de las primera parte.

\section{ESTUDIO FENOMENOLÓGICO DE UN PROBLEMA FÍSICO: LA CAÍDA LIBRE}

\section{El problema}

Las ciencias naturales no admiten leyes a priori, y sin embargo Galileo en su libro Consideraciones y Demostraciones Matemáticas sobre dos nuevas Ciencias demuestra a priori que los cuerpos más pesados (más grandes) no pueden caer en el vacío más rápido que los cuerpos menos pesados, por consiguiente, deben caer con la misma velocidad. Debe haber un error lógico en la demostración, ¿dónde?

\section{Comentario}

Nuestra línea de investigación se mueve dialécticamente entre la historia de una ciencia y la ciencia de la cual se hace historia. El problema que planteamos es histórico, pero nuestra preocupación primordial no es la histórica sino la científica, una preocupación que en ningún momento puede dejar de lado el contexto histórico en el cual se plantea el problema. En esta interacción dialéctica entre historia y ciencia se descubren parentescos, relaciones, contrastes y asociaciones que llevan a un refinamiento de los conceptos. Hay niveles de comprensión, los conceptos se refinan o se elaboran cuando se pasa de un nivel a otro. $Y$ aunque la historia no es el único método de refinamiento,

\footnotetext{
Profesor Asociado, Departamento de Sociales, Universidad Pedagógica Nacional Programa interinstitucional del Doctorado en Educación, Área Educación en Ciencias Naturales.
} 
tiene algunas ventajas sobre la simple resolución de problemas, como veremos a lo largo de la exposición.

\section{El relato histórico}

La física aristotélica sostenía que los cuerpos más pesados caen más rápido que los menos pesados, en el mismo medio. Un cuerpo de plomo, 10 veces más pesado que otro cuerpo de plomo, cae, en el aire, 10 veces más rápido. El interés de Galileo no es tanto refutar a Aristóteles como justificar su hipótesis de que todos los cuerpos caen con la misma velocidad en el vacío, independientemente de su peso y de su naturaleza, una aseveración completamente original y de una audacia enorme para su tiempo. Para exponer sus ideas, Galileo utiliza el diálogo, sus personajes simbolizan el pensamiento aristotélico: Simplicio el pensamiento libre de prejuicios Sagredo, el pensamiento revolucionario de la nueva ciencia: Salviati. En la reconstrucción del relato histórico seguimos el texto de las Consideraciones. Después de que Simplicio, a solicitud de Sagredo, ha expuesto la enseñanza aristotélica sobre la no existencia del vacío, en donde aparece la tesis aristotélica sobre la proporcionalidad de las velocidades y de los pesos, en un mismo medio, Sagredo toma la palabra para mostrar que muy a pesar de lo que afirma Aristóteles, la velocidad de caída de cuerpos diferentes, en un mismo medio, no es proporcional a los pesos.

Sagredo. Yo, sin embargo, señor Simplicio, que no he hecho la prueba, os aseguro que una bala de cañón que pese cien, doscientas o más libras, no aventajará ni siquiera en un palmo en su llegada al suelo, a una bala de mosquete de media libra, aunque la altura de la caída sea de doscientas brazas

Salviati. Sin recurrir a otras experiencias, podremos probar claramente, sin embargo, con una demostración breve y concluyente, que no es verdad que un móvil más pesado se mueva a más velocidad que un móvil más liviano, con tal de que ambos sean de la misma materia, como es el caso, sin duda, de aquellos de los que habla Aristóteles. Pero decidme antes, señor Simplicio, si admitís que a todo cuerpo pesado en caída libre le corresponda una velocidad determinada, de modo tal que no se pueda aumentar o disminuir a no ser que le hagamos violencia o le pongamos alguna resistencia.

Simplicio: Está fuera de toda duda que el mismo móvil en el mismo medio tiene una velocidad reglamentada y determinada por la naturaleza, la cual no podrá aumentarse a no ser por un impulso nuevo ni disminuirse si no es recurriendo a algo que la obstaculice y la retarde.

Salviati: Entonces, si nosotros tuviéramos dos móviles, cuyas velocidades naturales fuesen distintas, es evidente que si uniésemos ambos, el más rápido perdería velocidad por obra del más lento, mientras que éste aceleraría debido al más rápido. ¿Estáis de acuerdo con lo que acabo de decir?

Simplicio: Me parece que las cosas deben ciertamente, suceder así.

Salviati. Pero sí esto es así, y si es verdad, por otro lado, que una piedra grande se mueve, por ejemplo, con una velocidad de ocho grados y una piedra pequeña, con una velocidad de cuatro, si las unimos, el resultado de ambas, según lo dicho, será inferior a ocho grados de velocidad. Ahora bien, las dos piedras juntas dan por resultado una más grande que la primera que se movía con ocho grados de velocidad; de lo que se sigue que tal compuesto se moverá a más velocidad que la primera de las piedras sola, lo cual 
contradice vuestra hipótesis. Veis, pues, cómo suponiendo que el móvil más pesado se mueve a más velocidad que el que pesa menos, concluyo que el más pesado se mueve a menos velocidad mientras caen.

\section{Un experimento imaginario}

No es este el momento de discutir la importancia y el papel que desempeña el experimento imaginario y consiguientemente la argumentación imaginaria en el desarrollo de las ciencias. No se trata ciertamente de un simple artificio para suplir la experimentación real, se trata más bien de un paso intermedio, necesario, entre el mundo de las entidades puramente matemáticas y el mundo real. Su importancia es tal, que nos atreveríamos a afirmar que no hay experimento real que no se haya concebido antes como imaginario. Galileo está convencido de la existencia del vacío, pero la experimentación en el vacío es imposible para su tiempo. Esto no le impide imaginar el vacío y lo que podría pasar en la caída de dos cuerpos de la misma naturaleza y de diferente peso. La caída se debe a una fuerza inmanente al cuerpo, a su naturaleza, diría Aristóteles, por esta razón, así como al plomo en comparación con el cobre, por ejemplo, le corresponde un peso diferente por unidad de volumen (peso especifico diferente), así cabe suponer que al plomo le corresponde una velocidad natural de caída diferente a la del cobre. Dentro de este contexto se entiende que ja ley aristotélica, según la cual la velocidad de caída en un mismo medio es proporcional a los pesos, se refiere indudablemente a los cuerpos del mismo peso especifico. Galileo demostrará más adelante que todos los cuerpos, independientemente de su tamaño y de su peso específico, caen con la misma velocidad en el vacío. Esta demostración es propiamente el objetivo fundamental del texto de las Consideraciones que estamos estudiando, nosotros sin embargo elegimos arbitrariamente el argumento menos importan' te en la demostración, pero a nuestro modo de ver, bastante curioso. Hasta donde llegan nuestros conocimientos, se trata de un argumento que fue ignorado por los contemporáneos de Galileo, en particular por sus enemigos, que bien lo podrían haber aprovechado para ridiculizar sus pretensiones científicas. Galileo por otra parte, está absolutamente convencido de la legitimidad de su argumentación a prori, de lo contrario no se habría expuesto tan ingenuamente a los ataques de enemigos tan poderosos.

Antes de entrar al análisis del argumento, conviene reconstruir el experimento imaginario por medio de imágenes.

El cuerpo A es más pesado que el cuerpo B. El Cuerpo $C$ es la reunión de dos cuerpos idénticos a los cuerpos $A$ y $B$. Los dejamos caer en el vacío, es decir, en un medio 'infinitamente' sutil. Si el cuerpo B cae más lentamente que el cuerpo A, parece completamente evidente que el cuerpo $C$ caerá más lentamente que el cuerpo $A$, pues $B$ le resta velocidad. Habrá que hacer el experimento real? No es necesario. La experiencia común, cotidiana, nos dice que el corredor más lento le resta velocidad al más rápido si están unidos por una cuerda o por cualquier otro medio. Hasta aquí la argumentación parece completamente legítima. Pero Galileo continúa. El cuerpo $\mathrm{C}$ es más pesado (más grande) que $A$, por consiguiente debe caer más rápido que éste, de acuerdo con la hipótesis inicial de que los cuerpos más pesados caen más rápido. Llegamos así a la conclusión absurda de que un mismo cuerpo debe caer más rápido y más lento que otro. 


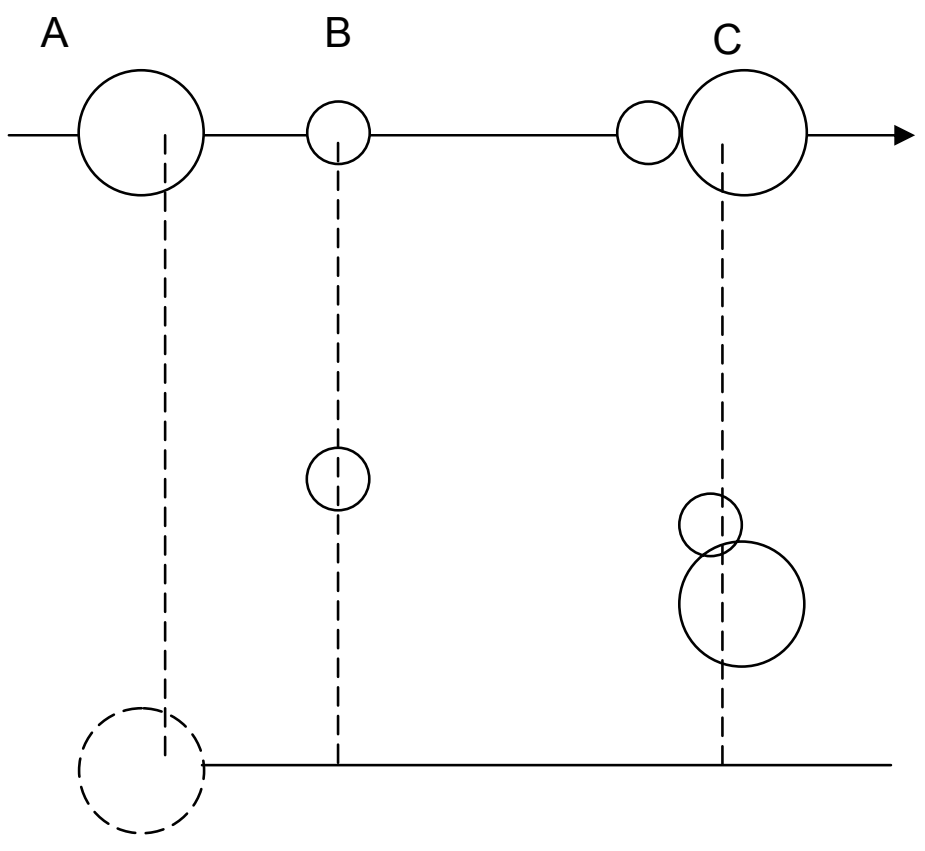

C cae más despacio que $A$, porque $B$ le resta velocidad. $C$ cae más rápido que $A$, porque es más pesado que éste. Un mismo cuerpo cae más despacio y más lento que otro.

La segunda parte de la demostración no nos convence tan fácilmente como la primera. Los dos cuerpos unidos por una cuerda no son un cuerpo, son dos, basta mirar la figura. Dos círculos no son un círculo! De acuerdo, pero en este caso la atención está dirigida a la forma, circulo, y no a la entidad física, piedra, por ejemplo. ¿No se puede decir con toda razón que dos piedras dan por resultado una piedra más grande? La forma (figura) influye ciertamente en la velocidad de caída en un medio, como lo sabia Aristóteles y el mismo Galileo. Una hoja de cobre no cae con la misma velocidad, en el aire, que un clavo de cobre. Pero en el vacío, donde no hay resistencia, la forma de la piedra no tiene por qué influir en la velocidad de la calda, ni siquiera, para los aristotélicos, que no admiten el vacío, pero que seguramente se lo pueden imaginar. Y si la forma no influye, ¿no habrá qué decir que dos piedras juntas forman una piedra más pesada? ¿No estamos convencidos? Acortemos la cuerda, despreciemos su peso, no dan dos piedras amarradas" por resultado una piedra más grande, o si prefiere, ¿más pesada? Y si es así, ¿no habrá que decir que caen más rápido que la mayor de ellas sola? La hipótesis nos lleva a una contradicción, es por consiguiente, falsa: los cuerpos más grandes (más pesados) no pueden caer más rápido que los cuerpos más pequeños, deben caer con la misma velocidad, en el vacío.

Supongamos ahora que el cuerpo A es de plomo y el cuerpo B de cobre. ¿Qué sucede? Que la argumentación se hace imposible, pues es evidente que la reunión de $A$ y de $\mathrm{B}$ no dan por resultado un cuerpo más grande, de la misma naturaleza de los dos cuerpos anteriores. Se entiende entonces por qué uno de los su puestos de la demostración es la igualdad de los pesos específicos.

La línea que estamos proponiendo supone que hay niveles de comprensión desde el más superficial al más profundo. Esta profundización se lleva a cabo de diferentes maneras, cuya formalización corresponde por supuesto a la investigación. Pero una de las 
formas de profundización es ciertamente la coordinación de unos conocimientos con otros en todo el sentido piagetiano del término. En el ensayo que estamos haciendo, el concepto de la caída libre entró en relación, cuando menos lo esperábamos, con otros conceptos, el de la aditividad del peso (el peso de A + el peso de B dan por resultado un peso mayor), el de la legitimidad o no legitimidad del apriorismo en física (se puede demostrar a prior que los cuerpos más pesados no pueden caer más rápido que los menos pesados?), la utilidad o no utilidad de la argumentación imaginaria, etc.

Finalmente, aprovechando el caso que nos ocupa, podemos salirnos por un momento del contexto histórico para plantear el problema en términos más actuales. El cuerpo $\mathrm{A}$ es más grande que $B$ y tiene además una cavidad exactamente igual a $B$. El cuerpo $C$ es igual a A, pero dentro de la cavidad lleva al cuerpo B. De acuerdo con la argumentación galileana, el cuerpo $C$ cae más despacio que $A$, porque $B$ le resta velocidad, y más rápido que $A$, porque es más grande. La conclusión no se puede aceptar, por lo tanto la suposición de que los cuerpos más pesados caen más rápido es falsa.

Ya no es solamente Simplicio quien se asombra, nosotros también. ¿Dónde está el error? Nuestros conocimientos físicos actuales son muchísimo más avanzados que los conocimientos del siglo XVII, la caída de los cuerpos no se debe a un ímpetu natural ni a una fuerza motriz inmanente a los cuerpos. Nadie tenía la menor noticia de lo que entendemos por masa inercial, a no ser Galileo, aunque de una forma todavía muy incipiente. Y sin embargo el problema planteado por esta Demostración al absurdo suscita nuestro interés, y lo que es más importante, nos hace confrontar nuestros conocimientos con los conocimientos de los fundadores de la Ciencia moderna.

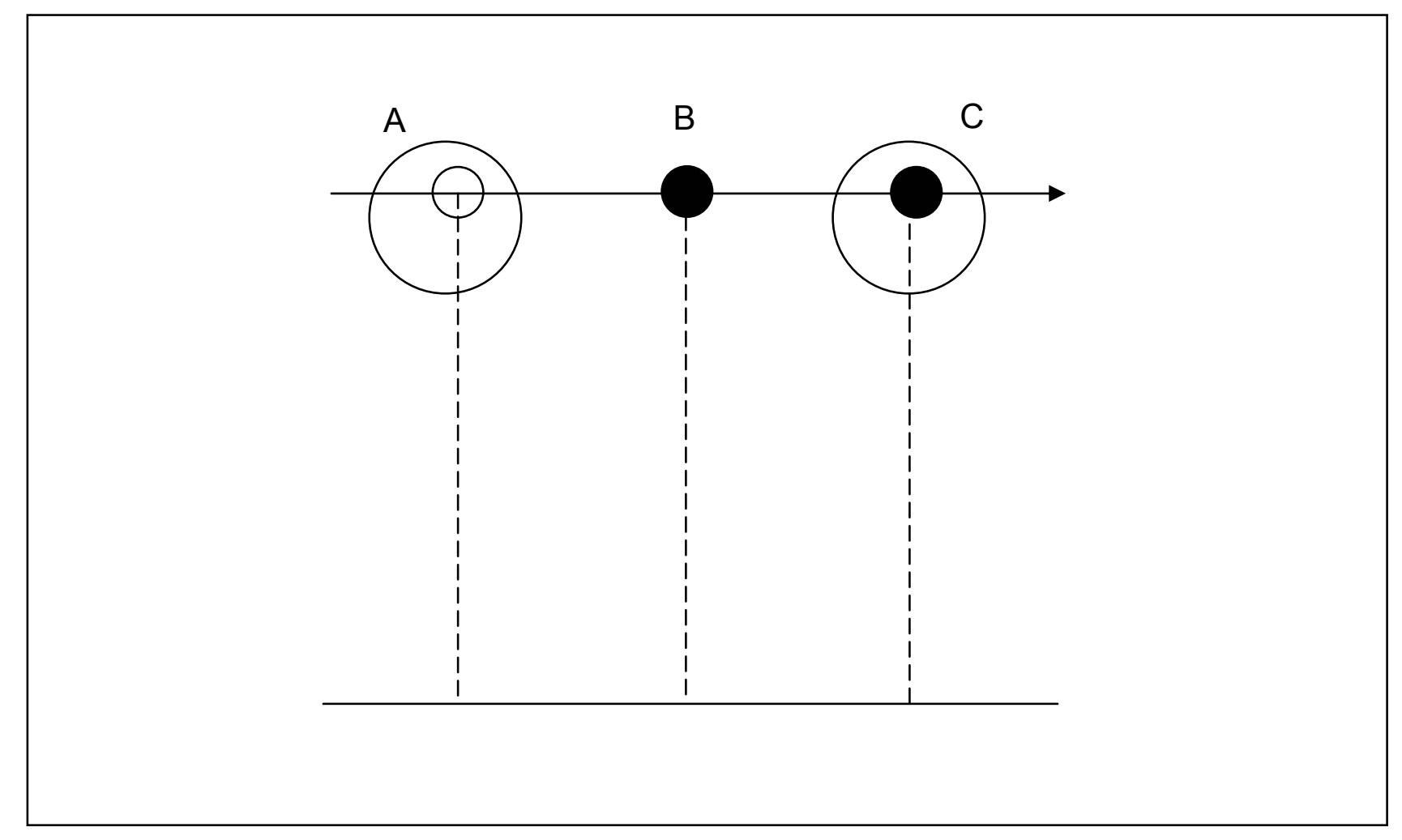


C cae más despacio que $A$ porque $B$ le resta velocidad, pero al mismo tiempo, o debe caer con más velocidad que A porque es más pesado. La suposición, por consiguiente debe ser falsa.

Una de las características de la línea es su preocupación pedagógica. Cómo resolverían nuestros estudiantes el problema en sus dos versiones, pues la versión que proponemos como moderna no hace más que confirmar la aparente fuerza del argumento galileano? No podemos responder hasta que no hayamos definido con claridad qué se entiende por nivel de comprensión y cómo es posible cuantificarlo. Se trata de todas maneras de un eventual campo de investigación.

\section{Análisis fenomenológico}

En toda actividad del conocimiento hay una doble intención, la directa y la refleja. En la actividad directa se aplican los conocimientos sin ninguna consideración ulterior sobre la forma como se aplican, en la consideración refleja se aplican los conocimientos y posteriormente el entendimiento intenta tomar conciencia del proceso mismo de conocimiento. Esta reflexión sobre su propia actividad es lo que generalmente se entiende por fenomenología. Sin entrar en discusiones, podemos considerar el análisis fenomenológico como una reconstrucción realizada por el científico, no por el filósofo o historiador, de la actividad científica, en la solución de un determinado problema, tomando la historia como base y orientadora de dicha reconstrucción.

Retomemos nuevamente el argumento, tal como lo presenta Galileo: Supongamos una piedra grande que cae con una velocidad de 8 grados y una piedra pequeña que cae con la velocidad de 4 grados. Si las unimos por medio de una cuerda: por una parte, la reunión de las dos caerá con una velocidad menor que 8 grados porque la menor le restará velocidad, y por otra, con una velocidad mayor, porque las dos piedras juntas dan por resultado una piedra mayor que la mayor de ellas

Desde el punto de vista filosófico, dos piedras son dos piedras y no una piedra más grande. Pero el problema no es filosófico sino físico, y cuando Galileo afirma que las dos piedras unidas por la cuerda dan por resultado una piedra más grande, lo que quiere decir es que dos piedras juntas pesan más que una de ellas por separado, una proposición que corresponde a la aditividad de los pesos, confirmada por la experimentación, y que nadie pone en duda. Habría una objeción, más aparente que real: los cuerpos no pesan, mientras caen, si por peso se entiende la presión sobre otro cuerpo, los platillos de una balanza por ejemplo. La reunión de los dos cuerpos no pesan más que el mayor de ellos, mientras están cayendo, por la simple razón de que ninguno de ellos pesa en el sentido expuesto. Galileo aprovecha la ocasión para disertar al respecto con una sencillez y claridad envidiable. Salviati acaba de exponer su argumento ad absurdum y Simplicio no sabe qué decir.

Simplicio Yo me encuentro completamente ofuscado, pues me parece que la piedra más pequeña unida a la mayor le da más peso, y no consigo explicarme cómo dándole más peso, no deba sumarie velocidad o, al menos, no disminuírsela.

Salviati: En este punto volvéis a cometer otro error, señor Simplicio, porque no es cierto que la piedra más pequeña aumenta el peso de la mayor.

Simplicio Esto está fuera de mi alcance! 
Salviati: Estará a vuestro alcance cuando os haga ver el equivoco en el que os encontráis metido.. Notad ante todo, que hay que distinguir los cuerpos graves en movimiento de los mismos en estado de reposo. Una piedra grande, colocada sobre una balanza, no solamente pesará más si se le añade otra piedra, sino que con adjuntarle sólo una brizna de estopa aumentaría su peso las ocho o diez onzas del peso de la estopa. Pero si, por el contrario, dejáis caer libremente, desde cierta altura, la piedra y, atada a ella, a la estopa, ¿creéis que el peso de la estopa, añadido al de la piedra, aceleraría el movimiento de ésta o más bien que lo que hará es disminuir su velocidad, sosteniéndola en parte? Sentimos sobre nuestras espaldas el peso de $\sim n$ objeto cuando intentamos oponernos al movimiento que se produciría si cayese, pero si somos nosotros mismos los que descendemos a la velocidad a la que caería de modo natural dicho cuerpo, ¿creéis que se nos echaría encima y sentiríamos su peso? No veis que esto seria como querer herir de una lanzada a alguien que huyera delante de nosotros a la misma velocidad o más rápido que nosotros mismos? Sacad la conclusión, por tanto, de que en la caída libre y natural, la piedra más pequeña no presiona con su peso a la mayor y, consecuentemente, no le añade peso alguno, como seria el caso en estado de reposo.

El concepto de caída libre (velocidad en la caída libre) entra en relación, cuando menos lo esperábamos, con el concepto de peso. Este ya no es, como lo suponíamos de una manera espontánea, una virtud o propiedad del cuerpo, el peso es una manifestación derivada, es la medida de la oposición al movimiento, de manera que donde no hay oposición, no hay medida. Para nosotros, hoy en día, la ausencia de peso en la caída libre no ofrece dificultad, precisamente porque ya sabemos que todos los cuerpos caen con la misma velocidad en el vacío, pero en el tiempo de Galileo las cosas no eran así, él mismo no puede suponer que los cuerpos en caída libre no pesan si no supone con anterioridad lo que está intentando demostrar. ¿Qué sucede si coloco un cuerpo sobre un resorte y los dejo caer? ¿Se comprime el resorte? La imaginación no puede decidir, habría que hacer el experimento. Evidentemente, si ambos, el cuerpo y el resorte, caen con la misma velocidad, el resorte no se comprimirá. De todas maneras, el argumento continúa en pié, aclarando que cuando se habla del peso de los cuerpos se está haciendo referencia al peso de estos en reposo. Galileo 0pta por hablar de tamaño, y así, en lugar de hablar del cuerpo más pesado, habla del cuerpo más grande, una sustitución completamente legítima si se piensa que a mayor tamaño, mayor peso, silos pesos específicos son iguales.

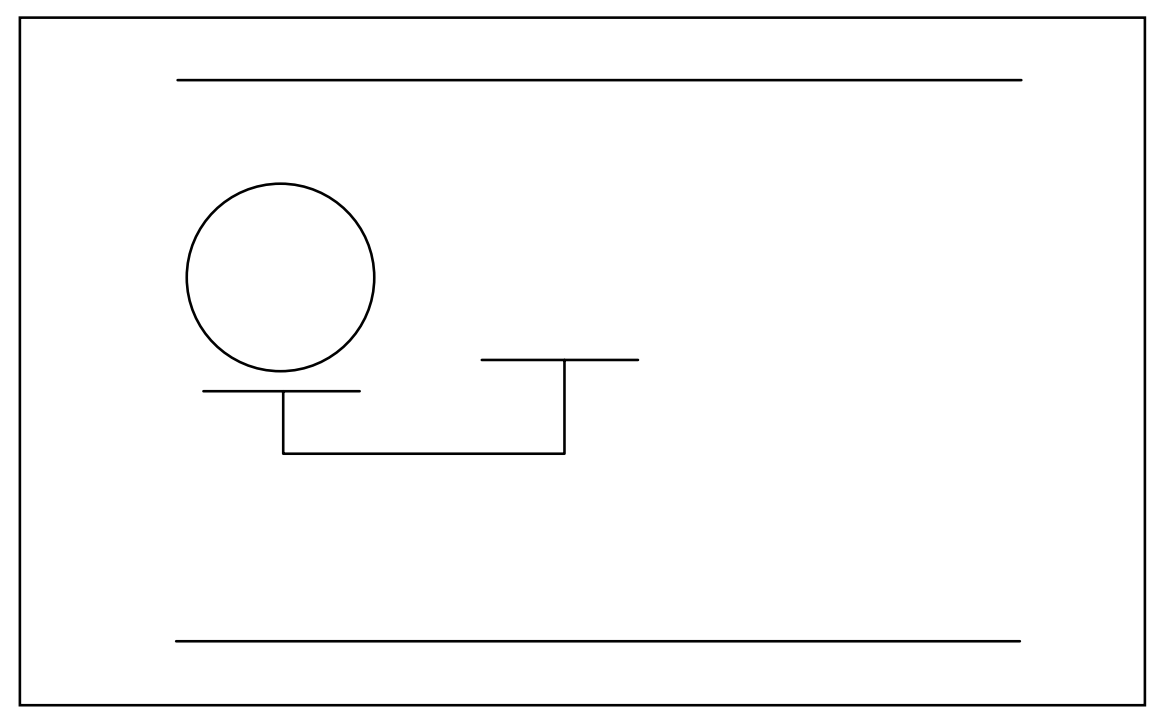


¿Pesan los cuerpos mientras caen? Depende. Si los cuerpos más pesados caen más rápido, entonces el más pesado presiona el menos pesado, y se detectaría un peso

La aclaración del concepto de peso es supremamente importante para la solución definitiva del problema. ¿Qué es, entonces, lo que produce el peso en los cuerpos en reposo? El ímpetu o virtud motriz del cuerpo. La fuerza motriz, diría, Juan Bautista Benedetti, predecesor y maestro intelectual de Galileo. El movimiento en la caída no se debe propiamente al peso, sino a la fuerza motriz, que suponemos proporcional al peso. Pero antes de entrar a la solución, creemos conveniente explicar la diferencia entre la historia pedagógica de las ciencias, como es el caso de la escuela de A. Koyré, y la fenomenología histórico-pedagógica de las ciencias, como es la pretensión de la línea que representamos.

\section{La explicación de A. Koyré}

A. Koyré, en su estudio "De motu gravium" de Galileo (Estudios de Historia del pensamiento científico, siglo XXI, editores) toca tangencialmente el argumento galileano. Su interés es más histórico que físico y por esta razón se esfuerza en sugerir algunas respuestas a Simplicio, dentro del contexto aristotélico, pero en ningún momento asume personalmente el problema, saliéndose, si fuese necesario del marco puramente histórico. Aquí encontramos una diferencia con el enfoque fenomenológico que pretendemos. Nuestro interés ya no es lo que podría haber respondido Simplicio, sino lo que podríamos responder nosotros, como físicos, utilizando en parte el contexto histórico y en parte el contexto actual. Es el pensamiento físico, no el histórico, el que asume el problema planteado por la historia, y es en este sentido que hablamos de análisis o de reconstrucción fenomenológica de la historia. Transcribimos a continuación el texto de A. Koyré:

\footnotetext{
"y en cuanto al experimento de Salviati, Simplicio habría podido responder igualmente que una gavilla de paja unida a una bala de cañón sigue siendo una gavilla de paja, como la bala de cañón sigue siendo una bala de cañón. $Y$ que si la gavilla cae lentamente cuando está sola, y la bala de cañón de prisa, es razonable, yen modo alguno contrario a la enseñanza de Aristóteles, admitir que, si se las uniera, la bala de cañón aceleraría el movimiento de la gavilla y ésta disminuiría el movimiento de la bala de cañón, aunque el peso del conjunto fuera mayor que el de los objetos que lo componen y sobre todo que el de la bala el conjunto compuesto por una gavilla de paja y una bala de cañón no es una bala de cañón más pesada. El conjunto no es un objeto natural. Además, igual que en su pretendida respuesta a Benedetti, Simplicio habría podido añadir que incluso si nos obstináramos, contrariamente al sentido común, a la razón -y a Aristóteles- en referir al conjunto lo que no vale más que para los componentes, deberíamos haber tenido en cuenta que al haber aumentado el volumen del conjunto -con relación a ja bala- mucho más que su peso, la resistencia al movimiento del conjunto aumenta también mucho más que su pesantez, y que es pues completamente normal -y una vez más, conforme a la dinámica de Aristóteles- que si disminuye la proporción entre fuerza moviente y resistencia, el movimiento, es decir, su velocidad, disminuya igualmente".
}

El argumento que pone A. koyré en boca de Simplicio es muy débil. Evidentemente la reunión de una gavilla de paja y de una bala de cañon no hacen una bala de cañon más pesada. Salviati podría haber contestado inmediatamente que su interlocutor olvida que tanto Aristóteles como Galileo hablan de cuerpos del mismo peso específico. Y si es así, no se ve cómo es posible que la reunión de un cuerpo de plomo de 100 libras (una bala de cañón) y otro cuerpo de plomo de 1 libra (una bala de mosquete), no den por resultado un cuerpo de plomo más pesado (una bala de cañón, por ejemplo). La velocidad de la caída no es una propiedad de la bala, sino del plomo. Una bala de cañón y una bala de 
mosquete, unidas por una cuerda, no hacen una bala de cañón más grande, pero si hacen un cuerpo más pesado (en reposo), que es lo que interesa en la demostración.

En cuanto al segundo argumento: al aumentar el peso, aumenta también la resistencia y por consiguiente no se puede concluir, sin más, que la reunión de los dos cuerpos cae más rápido que el mayor de ellos, Salviati podría haber respondido con toda razón que la resistencia del medio no aumenta en el caso de los dos cuerpos unidos por una cuerda ( $y$ en este caso es A. Koyré quien hace una suposición falsa). Cuando se supone que el cuerpo mayor cae con una velocidad de 8 grados y el menor con una velocidad de 4 grados, se está incluyendo la resistencia del medio, y esta resistencia sigue siendo la misma en el caso de la reunión de los dos cuerpos (por medio de una cuerda). Otra cosa seria, si en lugar de unirlos, se fusionan en uno solo. De todas maneras, la discusión carece de interés para Galileo, pues su argumentación imaginaria se lleva a cabo en el vacío.

\section{J. B. Benedetti}

¿Caen dos cuerpos iguales, unidos por una cuerda, más rápido que por separado? Galileo no es el primero que se plantea dicha pregunta, antes de él lo hizo Juan Bautista Benedetti (diversas especulaciones matemáticas y físicas), para llegar a la conclusión de que no hay ninguna razón en favor de la respuesta afirmativa. Pero si no hay ninguna razón en favor, tampoco hay ninguna razón en contra. Planteemos la pregunta de otra forma, una poco diferente: ¿no son dos cuerpos unidos por una cuerda, más pesados que uno solo de ellos, en reposo, por supuesto? Y si es así, ¿no habría que concluir, a pesar de la opinión de J. B. Benedetti, que caen más rápido? ¿Se dirá acaso que los cuerpos, juntos o separados, no pesan, mientras caen? Aquí radica precisamente el meollo del problema. Si no pesan más juntos que separados es porque caen con la misma velocidad, lo que pretendemos demostrar. Además, el argumento no se basa sobre el peso o no peso de los cuerpos mientras caen, sino sobre el peso en reposo. El presupuesto es por consiguiente: si los cuerpos que pesan más en reposo, caen más rápido, entonces se seguiría el absurdo de que un mismo cuerpo caería más rápido y menos rápido que otro. Como se explicó anteriormente, para evitar esta aparente objeción, galileo prefiere hablar de cuerpos más grandes y más pequeños, conservando el argumento toda su fuerza. Dos caballos unidos por la brida no corren más rápido. De acuerdo, a no ser que la velocidad dependa del peso de los caballos. Dos personas cogidas de la mano no caen más rápido que una sola! De acuerdo, a no ser que la velocidad de caída dependa del peso de las personas, en reposo, por supuesto.

La discusión que estamos haciendo parece bastante trivial, no era sin duda para los contemporáneos de Galileo. Los textos de física presentan la caída libre como una simple consecuencia de la igualdad de la aceleración gravitacional para todos los cuerpos, independientemente de su peso (propiamente, de su masa inercial). Pero, ¿cómo es posible que dos cuerpos de pesos diferentes caigan con la misma aceleración si se supone que la aceleración es proporcional al peso (fuerza motriz)? Hoy en día, conocemos la respuesta, la aceleración no es solamente proporcional al peso, sino inversamente proporcional a la masa inercial del cuerpo. La dificultad en la solución del problema que estamos analizando radica fundamentalmente en la ausencia del concepto de masa inercial. Si la fuerza motriz no es una fuerza externa sino una fuerza inmanente al cuerpo, de acuerdo con la tradición aristotélica, entonces es supremamente difícil imaginar que el mismo cuerpo sea la causa de su movimiento y de la oposición a éste. La caída libre sigue siendo para Galileo, a pesar de su aversión al aristotelismo, un 
movimiento natural, no violento, y no se ve cómo en un movimiento natural puedan coexistir el ímpetu natural al movimiento y su oposición, representada por la masa inercial.

\section{La solución definitiva}

\section{¿En dónde está el error de Galileo?}

Supongamos cuatro cuerpos, A, 8, 0, D. O es la reunión de A y 8; D es un cuerpo que pesa en reposo lo mismo que $O$. Representemos las fuerzas motrices, proporcionales a los pesos, mediante flechas, como indica la figura. De acuerdo con el argumento galileano, el cuerpo $O$ debe caer con menor velocidad que el cuerpo $A$, porque 8 le resta velocidad y el cuerpo $D$ con mayor velocidad que $A$, porque es más grande (mayor fuerza motriz).

El error está en haber utilizado un mismo término con dos significados diferentes: cuando afirma que la reunión de $A$ y de 8 debe caer más lentamente que $A$, está utilizando como modelo el cuerpo $O$, en donde las fuerzas motrices permanecen separadas, cuando afirma que la reunión de $A$ y de $B$ debe caer mas rápido que $A$, está utilizando el modelo $D$, en donde las fuerzas motrices dan por resultado una fuerza mayor.

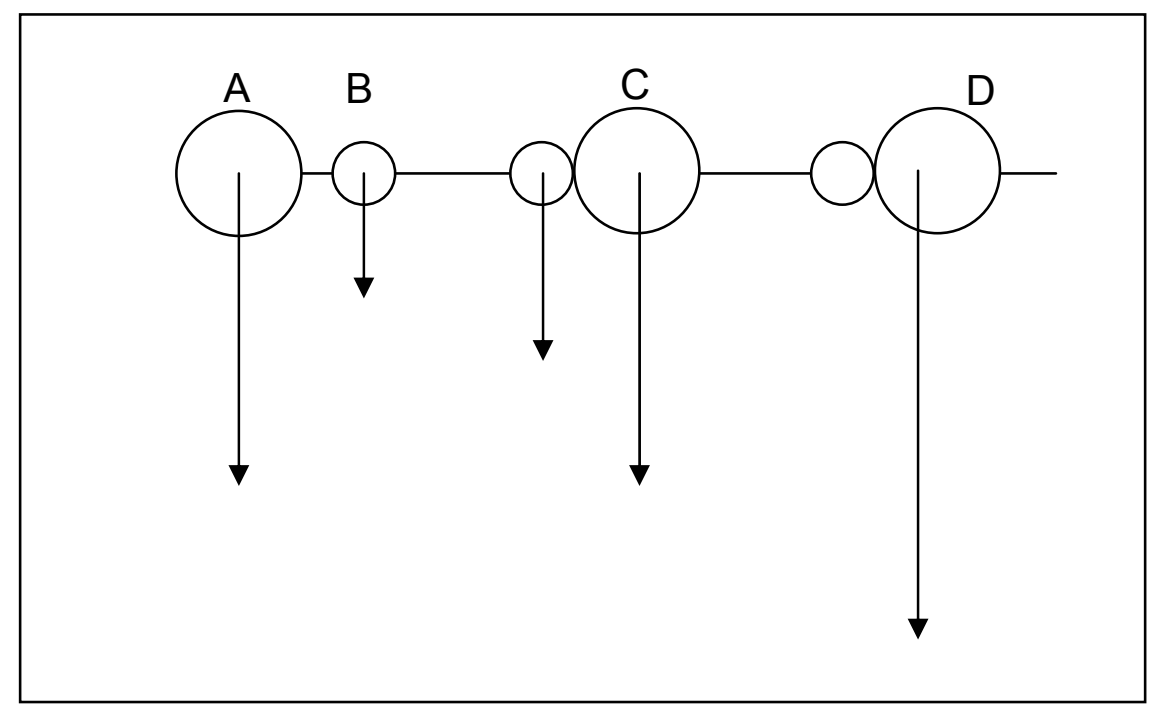

Galileo utiliza el modelo $O$ en una parte de su demostración, luego el modelo D en la otra parte. El error lógico está en haber utilizado un mismo término con dos significados distintos.

¿Dan dos piedras unidas por una cuerda por resultado una piedra más pesada (en reposo)? La respuesta está confirmada por la experiencia y no admite dudas, si. El origen de las dificultades está en la confusión entre peso y fuerza motriz. La fuerza motriz es la causa del peso en reposo y de la velocidad de caída y es además proporcional al peso en reposo. Todo esto lo sabían los contemporáneos de Galileo y él mismo. Aristóteles lo habría aceptado sin objeciones. Dos piedras dan por resultado una piedra más pesada, pero no, necesariamente, una piedra con mayor fuerza motriz.

Hemos encontrado el origen del error lógico en la argumentación galileana, pero nuestro interés va más allá de lo puramente lógico. ¿Cuál de los dos casos se ajusta más a la realidad, el $\mathrm{O}$ (las fuerzas motrices permanecen separadas) o el D (las fuerzas 
motrices se suman)? La respuesta está más allá del experimento imaginario, es necesario recurrir al experimento real. Ahora bien, ¿qué nos dice el experimento real? Que los cuerpos caen con la misma velocidad en el vacío. Si es así, Entonces los casos $\mathrm{O}$ y $\mathrm{D}$ son equivalentes, y esto no es posible si la única causa del movimiento es la fuerza motriz. Debe haber otra causa: la inercia.

La discusión del problema galileano nos ha llevado finalmente a una pregunta crucial, ¿se suman las fuerzas motrices o permanecen separadas? Nuestra respuesta, a 4 siglos de distancia, y en posesión del concepto de inercia, es perfectamente clara y coherente: los dos casos son completamente equivalentes. La velocidad de caída del cuerpo $\mathrm{O}$ es igual a la velocidad de caída del cuerpo $D$, pues lo que determina la velocidad de caída no es solamente la fuerza motriz sino la relación fuerza motriz-inercia, y esta relación es igual para todos los cuerpos y se denomina aceleración. Es Galileo, precisamente, el primero en formular el principio de inercia, aunque de una manera inexacta : los cuerpos tienden a conservar su estado de movimiento uniforme circular, no rectilíneo, como será la formulación definitiva de Newton.

$$
\text { Si } \frac{F_{A}}{m_{A}}=\frac{F_{B}{ }^{1}}{m_{B}} \text { entonces: }
$$

$$
\frac{F_{A}}{m_{A}}=\frac{F_{B}}{m_{B}}=\frac{F_{A}+F_{B}}{m_{A}+m_{B}}
$$

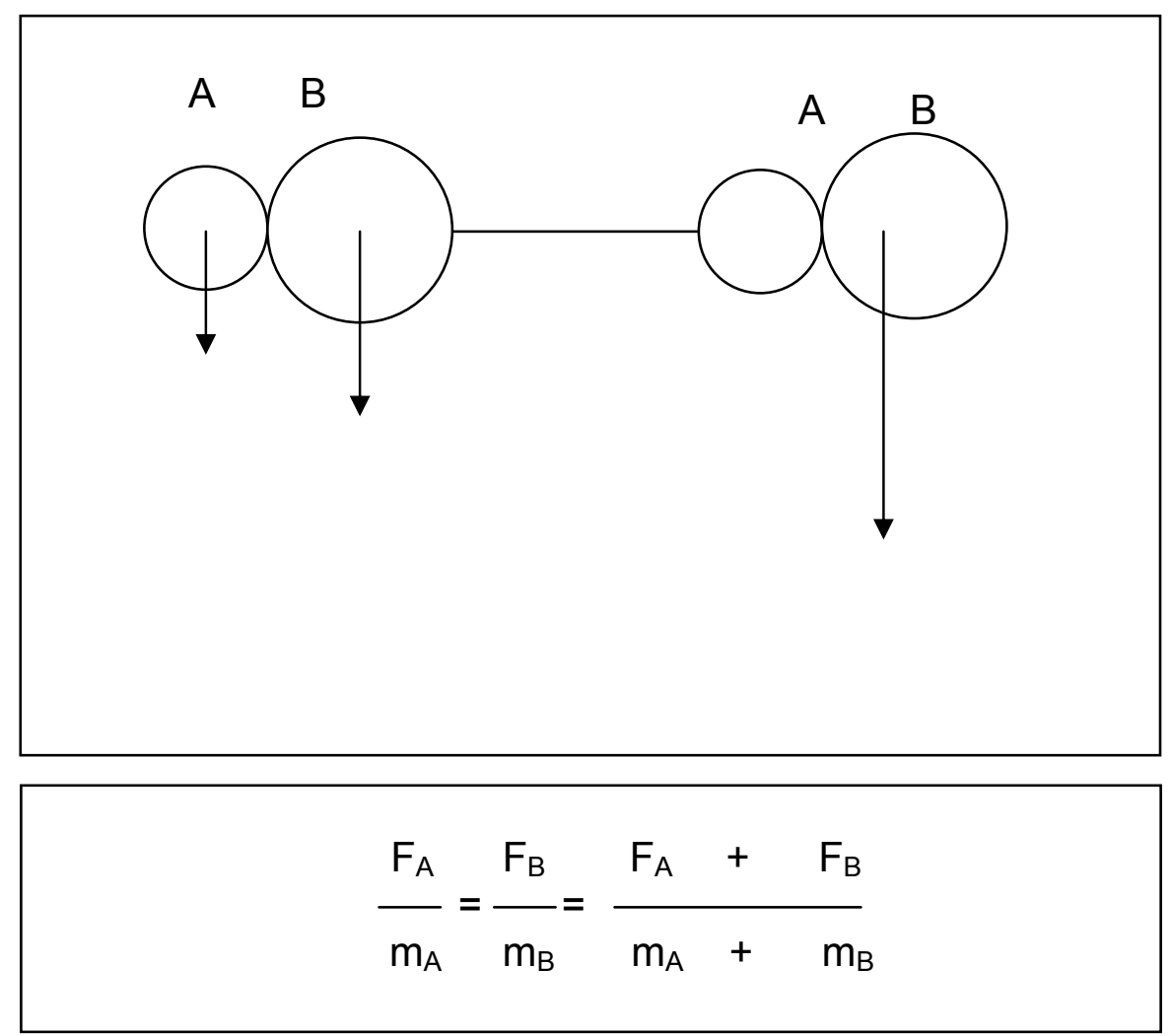


y por consiguiente, ya que las velocidades finales son proporcionales a las fuerzas motrices e inversamente proporcionales a las masas inerciales, que a su vez son proporcionales a las masas gravitacionales, la velocidad de A y de 8, cada una por separado, es igual a la velocidad de los dos cuerpos juntos:

$\mathrm{V}_{\mathrm{A}}=\mathrm{V}_{\mathrm{B}}=\mathrm{V}_{\mathrm{C}}$

Es decir: las fuerzas motrices pueden considerarse por separado o sumadas, el resultado es el mismo.

\section{Epilogo}

J. B. Benedetti había demostrado", a su parecer, que los cuerpos de peso especifico igual caen con la misma velocidad en el vacío, independientemente de su tamaño, y que los cuerpos de peso especifico diferente caían con velocidades proporcionales a dichos pesos. Se trata de una demostración muy curiosa desde el punto de vista histórico y físico, que bien merecería una investigación fenomenológica semejante a la que hemos realizado en estas páginas. Pero Galileo va más allá, no solamente los cuerpos del mismo peso específico caen con la misma velocidad, sino todos los cuerpos, independientemente de su tamaño y peso especifico. Una proposición inaudita, que coloca a Galileo dentro de los físicos más grandes de todos los tiempos. Realmente Galileo podría haber prescindido de la demostración a priori que hemos estudiado, sin embargo, no lo hizo ¿Por qué? Es difícil creer que se tratara de un simple artificio para suscitar la perplejidad de sus lectores, si se tiene en cuenta, como ya lo dijimos, que sus enemigos, además de numerosos, eran muy poderosos. Hay además otro hecho histórico, ninguno de sus contemporáneos se ocupó del argumento, posiblemente porque lo encontraron irrefutable dentro de los medios teóricos de que disponían. Nos atrevemos a pensar que Galileo estaba convencido de la legitimidad de su argumentación, aunque ésta ocupa un puesto secundario en la demostración de su hipótesis fundamental de que todos los cuerpos caen con la misma velocidad en el vacío, aunque sean de pesos específicos diferentes.

¿Por qué escogimos esta demostración y no la otra? Porque queríamos mostrar que la historia ofrece innumerables oportunidades de elaboración de conceptos. Sólo hay una condición: ir a las fuentes originales de autores científicamente relevantes.

\section{MARCO TEÓRICO DE LA LINEA}

\section{Los conceptos no se trasmiten, se elaboran}

Los conceptos no se trasmiten como se trasmite, por ejemplo, una información. Los conceptos se elaboran, tanto por parte de quien los aprende como de quien los enseña. El aprendizaje es necesariamente un proceso de elaboración por parte del alumno o estudiante, como la enseñanza es así mismo un proceso de elaboración dirigido al alumno. Son dos procesos paralelos, pero diferentes. Evidentemente el objetivo de la enseñanza es el aprendizaje y por consiguiente son las leyes de la elaboración de los conceptos en el alumnos las que rigen el proceso de enseñanza y no al contrario. Entre elaboración y construcción hay una diferencia de matiz que es importante resaltar. La construcción hace referencia a las estructuras o esquemas generales, la elaboración, además, a la consolidación y ornato de esas estructuras. 


\section{El estructuralismo y el conductismo}

Los conceptos son sistemas de operaciones formales y estos sistemas tienen una génesis perfectamente definida a partir de los esquemas más primitivos de las acciones reflejas. Estos sistemas de operaciones tienen las características de los grupos matemático y de las clases lógicas (identidad, asociatividad, transitividad, reversibilidad, etc.). Los conceptos se construyen según determinadas leyes, este es el gran aporte de Piaget y de sus seguidores dentro de la psicología de la inteligencia. Dentro de esta perspectiva abundan los estudios estructuralistas sobre la génesis del número, sobre la formación de los conceptos de masa, peso, velocidad, tiempo, etc. Pero el constructivismo piagetiano no va más allá de la constatación de estas leyes de construcción que dependen en último término de las etapas de maduración sico-biológicas del individuo. La sicología genética se ocupa por lo tanto de la construcción espontánea de los conceptos, no de su elaboración intencional con vistas a una explicación. Se trata por tanto de dos aproximaciones diferentes y no excluyentes: La aproximación sicológica de los estructuralistas y las aproximación "fenomenológica" que proponemos en esta línea de Investigación y cuya justificación se hará a lo largo de esta exposición.

Para la escuela conductista o skinneriana el concepto no es más que una conducta o comportamiento determinado. El individuo que resuelve exitosa-mente problemas que suponen el concepto de aceleración, tiene dicho concepto, y este concepto se perfecciona en cada nueva solución exitosa. La elaboración interna se lleva a cabo a través de la ejecución externa de acciones apropiadas. La elaboración externa o magisterial se lleva a cabo a través de la programación de la secuencia de acciones y de sus correspondientes refuerzos. Las aproximaciones de Skinner y de Piaget son en último término aproximaciones sicológicas, desde fuera de la actividad científica como tal, y no aproximaciones interiores a dicha actividad. Evidentemente no es lo mismo resolver un problema de matemáticas que reconstruir los pasos que uno mismo ha llevado a cabo en la solución, ambas son actividades matemáticas, la primera directa y la segunda refleja.

\section{La aproximación fenomenológica}

El término fenomenología, que nació en un contexto puramente filosófico a partir de Husserl, tiene la propiedad de expresar el carácter inmanente de cualquier aproximación. La investigación fenomenológica de los conceptos científicos debe hacerse desde dentro de la ciencia misma. En una palabra, no se trata de hacer sicología de la física, sino física de la física o lo que es lo mismo fenomenología de la física. La fenomenología es la actividad por medio de la cual el pensamiento descubre o intenta descubrir el propio proceso de razonamiento con sus aciertos, errores, suposiciones equivocadas, etc.

\section{Fenomenología e historia}

La fenomenología es eminentemente personal, nadie puede pensar por otro, ni mucho menos reconstruir el proceso de pensamiento de otro. Sin embargo, es posible una fenomenología de la historia de las ideas científicas en la medida en que se acepte que la ciencia es producto histórico y que hacer ciencia es en gran medida apropiarse la tradición histórica. No otra cosa hace el físico cuando por ejemplo utiliza el concepto de conservación del impulso en la solución de un problema determinado. El concepto es suyo, pero el concepto es así mismo el resultado de un largo proceso histórico de elaboración que el científico toma como propio. Lo que se afirma de los conceptos físicos se afirma también de los conceptos químicos y biológicos: son elaborados históricamente y pueden ser estudiados fenomenológicamente, es decir, por el físico, químico o biólogo, 
según el caso y como objetos de física, química o biología. Ambos aspectos son importantes, pues así como el sicólogo puede hablar de física, así el físico puede hablar de psicología. Nuestra línea de investigación se define entonces como una investigación fenomenológica de la elaboración de algunos conceptos científicos a partir del estudio de la historia de la disciplina en cuestión y realizada desde dentro de disciplina. En el caso del principio de conservación del impulso, la investigación debe ser realizada por un físico con conceptos físicos y tomando como base de sus reflexiones la elaboración histórica de dicho concepto. Hay por consiguiente dos elaboraciones paralelas, la histórica y la fenomenológica, la histórica permanece exterior a la física, al fin y al cabo es historia de la física, la fenomenológica entra dentro del pensamiento físico, examinando desde dicha perspectiva el contenido histórico. Históricamente el concepto de impulso nace con Descartes (el producto de masa y velocidad) como contrapuesto al principio de conservación del reposo. Dios conserva en el universo tanta cantidad de movimiento y de reposo cuanta le comunicó en el primer instante de la creación. Cuando un cuerpo mueve a otro, le comunica impulso, pero como la cantidad de impulso permanece constante en el universo hay que concluir que el impulso que recibe un cuerpo lo pierde el otro y viceversa. De aquí se siguen 7 reglas del choque, una de las cuales dice que un cuerpo más pequeño que otro (menos cantidad de materia), por pequeña que sea la diferencia y por grande que sea su velocidad no podrá mover a otro cuerpo mayor en reposo. Es el inicio de una polémica de más de 30 años, cuyos protagonistas principales son Malebranche y Leibniz y que no es nuestro propósito actual reconstruir. El principio de la conservación tiene una larga e interesantísima elaboración que no superará la aproximación histórica mientras no suscite preguntas físicas para ser resueltas por físicos, y es en este esfuerzo donde se elabora fenomenológicamente el principio de conservación del impulso. Es como si se tratara, en una primera aproximación, de hacer física con problemas antiguos, para mejor comprender nuestros conceptos actuales. En la primera parte de esta exposición dimos un ejemplo de a elaboración fenomenológica del concepto de caída libre.

\section{La intención de la elaboración histórico-fenomenológica es pedagógica}

La intención de las diferentes historias de las ciencias varia de un autor a otro, sin embargo es posible mencionar cuatro intenciones bien definidas:

1. La confirmación de una teoría sobre la elaboración o construcción de los conceptos en el niño como seria el caso de las epistemologías genéticas de Piaget.

2. La elaboración del documento histórico de las ciencias, sin intenciones filosóficas o pedagógicas, como sería el caso de A Dabro en The Rise of the New Physics o de H. A. Klein en The Science of Measurement (R. Dugas, A History of Mechanics, etc.).

3. La reflexión sobre el fenómeno de la ciencia, bajo el supuesto de que la ciencia es un producto histórico y el conocimiento de su historia no sólo contribuye a su esclarecimiento sino que es una condición necesaria de cualquier tipo de epistemología científica. Dentro de este grupo de investigadores cabria poner a T. Kuhn (las estructuras de las revoluciones científicas, el surgimiento de lo nuevo), Eduardo Nicole (los principios de las ciencias), etc.

4. El descubrimiento de elementos pedagógicos que de alguna forma contribuyan a la enseñanza de las ciencias, como seria el caso de Koyré, Bachelard, etc. 
Nuestra línea de investigación se inscribe dentro de la escuela francesa liderada por A. Koyré con acentuación del aspecto fenomenológico. G. Bachelard pretende hacer un psicoanálisis de la ideas científicas, a partir de la historia y con intenciones pedagógicas; nosotros pretendemos hacer una fenomenología, no ya de las ideas científicas en general, sino de las ideas (o conceptos) de una disciplina como la Física ( Química, Biología). Mientras que el sicoanálisis busca remover los "obstáculos epistemológicos, la fenomenología busca reconstruir los procesos ocultos que llevan a la construcción de los conceptos. M. de Foucault prefiere hablar de arqueología, en donde la preocupación no son los obstáculos, ni la elaboración de los conceptos, sino los estratos sobre los cuales se construyen las ciencias.

\section{Requisitos}

Los conceptos científicos, como productos que son, pueden ser objeto de investigación desde multitud de perspectivas, como, por ejemplo, la de la sicología de la inteligencia o la de la inteligencia artificial. Nuestra perspectiva es exclusivamente científica. El principio de conservación de la masa puede ser estudiado por un sicólogo de la inteligencia, como de hecho lo hizo Piaget, o un historiador de las ciencias, como Dugas, o por un físico. Cuando decimos que nuestra perspectiva es primordialmente científica estamos escogiendo la última alternativa, la del físico, la del biólogo o la del químico, según el caso. Por esta razón el requisito, sine qua non, de la línea es el conocimiento profesional de una de las tres áreas que hemos mencionado.

\section{Metodología}

La metodología tiene inicialmente los siguientes pasos

\section{Definición del problema}

La investigación se debe llevar a cabo alrededor de uno o varios problemas claramente definidos. No se admiten formulaciones generales, como. Desarrollo histórico del concepto de calor o equivalentes.

\section{Lectura actual}

Una vez que se ha planteado el problema se intenta resolver con todos los medios disponibles que proporciona la ciencia o ciencias implicadas. Dentro de este marco teórico actual' se debe moverla interpretación fenomenológica, en una especie de interacción entre lo viejo y lo nuevo.

\section{Lectura histórica}

Después de la lectura actual del problema se pasa a la lectura histórica del mismo. Para el efecto se recoge toda la información histórica posible, procurando acudir a las fuentes.

\section{Reconstrucción fenomenológica}

El investigador debe moverse continuamente dentro de las dos lecturas mencionadas. Un ejercicio que no es fácil, pero que constituye el aspecto original de a línea en comparación con otras aproximaciones históricas semejantes. En este proceso de interacción dialéctica entre la historia y la ciencia, la ciencia ocupa el primer lugar. El oficio del investigador no es el de un historiador que hace ciencia, sino de un científico que hace historia, con la posibilidad siempre presente de salirse del contexto histórico para disertar sobre las implicaciones científicas del problema que está estudiando. 


\section{BIBLIOGRAFIA}

\section{Las fuentes}

Todos los científicos que han aportado algo al desarrollo de las ciencias son objeto posible de consulta. Más aún, la bibliografía fundamental, como se explicó con anterioridad está constituida por las fuentes en cada una de las áreas elegidas, fisica, química o biología

2. Historiadores

BACHELARD G. Etude sur Levolutíon dun probleme de Physique, Vrin. Paris, 1973.

CROSLAND M. P. Historical Studies in the Language of Chemistry, 1978.

DABRO A. The evoiution of Scientific Thought, Dover, N.Y., 1950.

D’ABRO A. The Rise of the New Physics, Dover, N.Y., 1951.

DUGAS R. A History of Mechanics, Dover, N.Y., 1988.

DUHEM P. To save the Phenomena, The University of Chicago Press, 1969.

HEITLER W., The Quantum Theory of Radiation, Dover Publications, N.Y., 1954.

JAFFE B., Crucibles: The story of Chemistry, Dover Publications, N.Y., 1976.

KLEIN A. The Science of Measurement, Dover, N.Y., 1974.

KLEINER A. Physik im 19. Jahrhundert, Darmstadt, 1980.

KOYRE A. Estudios de Historia del Pensamiento Científico, siglo XXI editores, 978.

KOYRE A. La revolution astronomique, Histoire de la Pensée III, Hermann, 1965

KOYRC A. Newtonian Studies, The university of Chicago Press, 1965.

WHITTAKER SE. A History of the Theories of Aether and Electricity, Dover, N.Y., 1989. 